\title{
The Challenge of Maintaining a Healthy Microbiome during Long-Duration Space Missions
}

\author{
Alexander A. Voorhies and Hernan A. Lorenzi * \\ Department of Infectious Disease, J. Craig Venter Institute, Rockville, MD, USA
}

Astronauts will face a host of challenges on long-duration space missions like a human expedition to Mars, including the difficulty of maintaining a balanced and healthy microbiome. The human microbiome is the collection of all microorganisms residing in and on a human host, and it plays an essential role in keeping humans healthy. However, imbalances in the microbiome have also been linked to many human diseases. Space travel has been shown to alter the microbiome of astronauts in ways that are not yet completely understood. Here we review past and current microbiology and microbiome

OPEN ACCESS

Edited by:

Jack Van Loon,

VU University, Netherlands

Reviewed by:

Vyacheslav Ivanovich Dokuchaev,

Institute for Nuclear Research of the

Russian Academy of Sciences, Russia Wen-Biao Han

Astronomical Observatory, China Michael Shannon Roberts,

Center for the Advancement of

Science in Space (CASIS), USA

*Correspondence. Hernan A. Lorenzi hlorenzi@jcvi.org

Specialty section:

This article was submitted to Cosmology,

a section of the journa Frontiers in Astronomy and Space

Sciences

Received: 15 March 2016 Accepted: 08 July 2016

Published: 22 July 2016

Citation: Voorhies AA and Lorenzi HA (2016) The Challenge of Maintaining a Healthy Microbiome during Long-Duration Space Missions. Front. Astron. Space Sci. 3:23. doi: 10.3389/fspas.2016.00023 research with the aim of determining the extent of change to the human microbiome caused by space travel and implications for astronaut health. We also address several challenges that will need to be overcome in order to facilitate long-duration human exploration missions. These challenges include maintaining environmental conditions that favor healthy microbiomes, controlling the microbial organisms astronauts are exposed to, the impact of galactic cosmic radiation on the microbiome, and medical interventions that can potentially damage the microbiome.

Keywords: microbiome, space flight, Mars, astronaut, International Space Station

\section{INTRODUCTION}

A manned mission to Mars will present a host of challenges for the astronauts making the long journey, including the difficulty of maintaining a healthy microbiome. The human microbiome is the collection of all microorganisms residing in and on the human body, and includes bacteria, archaea, single-celled eukaryotes and viruses. Our microbiome helps us break down food, protects us from infection and bolsters our immune system; we could not live without it. However, imbalances in commensal microbiota have been associated with a wide range of medical conditions, from irritable bowel syndrome (Saulnier et al., 2011) to diabetes (Giongo et al., 2011), different types of cancer (Schwabe and Jobin, 2013), and even depression (Luna and Foster, 2014; Figure 1). Frequently, the transition from a healthy to a disease state is accompanied by inflammation and a reduction in microbial diversity. Stressful conditions, like those typically found during a space mission, including cosmic radiation (Packey and Ciorba, 2010; Lam et al., 2012) and microgravity (Nickerson et al., 2004), have been shown to promote microbial dysbiosis and changes in bacterial physiology (Nickerson et al., 2000; Wilson et al., 2008; Bailey et al., 2011; Castro et al., 2011). Therefore, keeping a healthy microbiome during the 1000+ days of a manned mission to Mars will not only be challenging, but also imperative for crew health and mission success.

Maintaining a healthy and diverse microbiome on Earth is something most people do not think about. You take in microbes when you breathe, when you eat food, or even wipe your mouth. You encounter microbes when you shower, use your keyboard, or kiss your children. 


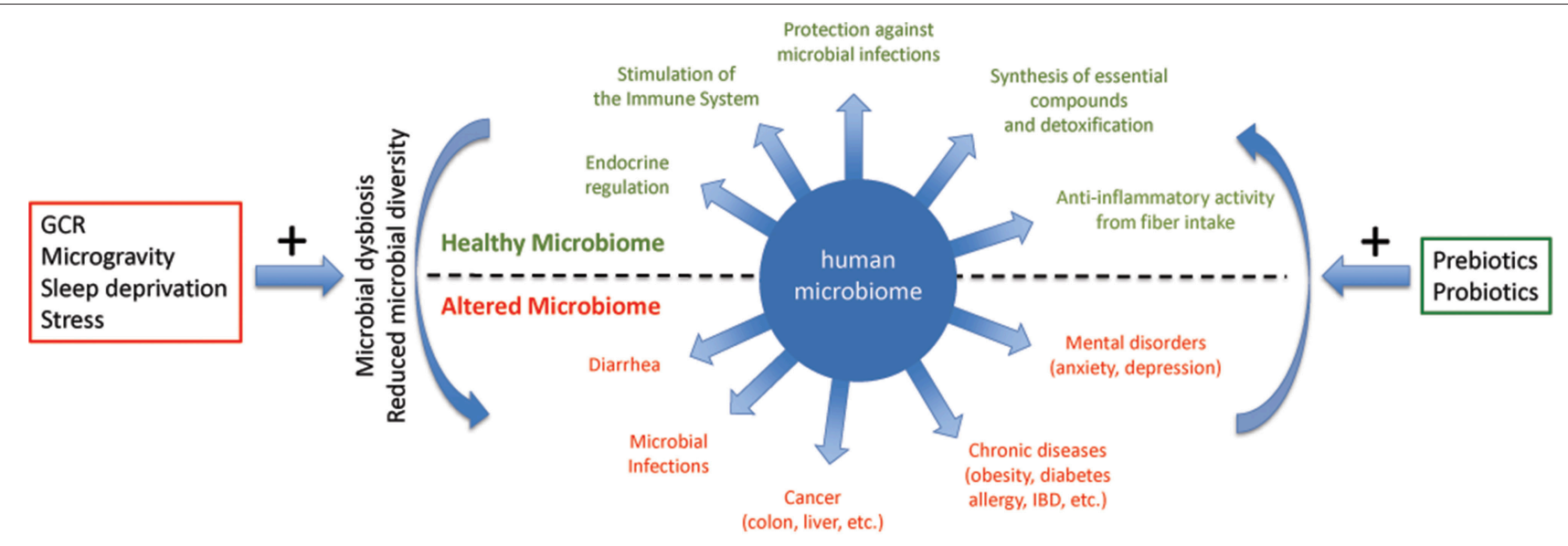

FIGURE 1 | Effects of space travel on the human microbiome. Stressors associated with space travel (red box) may disrupt the composition of the human microbiome causing dysbiosis. Microbial imbalance could result in the loss of some of its beneficial effects on human health (green) increasing the risk of astronauts of contracting infection or developing human diseases (red). Prophylactic treatments with prebiotics or probiotics (green box) could be used to counterbalance the detrimental effects of space travel on the astronauts' microbiome.

We are constantly replenishing our microbiomes with a fresh collection of new organisms that help us maintain a diverse and healthy collection of microbes (Costello et al., 2009; Consortium, 2012; David et al., 2014a,b). Taking in a greater diversity of organisms is usually correlated with a healthier microbiome, and chronic health issues like asthma have even been correlated with population shifts from rural to urban living spaces, and the subsequent reduction of exposure to a diverse collection of microorganisms in urban environments (Hanski et al., 2012).

Not all of the organisms we incorporate from the environment are beneficial to humans. Some microorganisms are opportunistic pathogens, a term that describes organisms that are healthy or neutral at low concentrations, but become pathogens when their populations increase or under certain favorable environmental conditions, such as an altered host microbiome or a weakened immune system. Most opportunistic pathogens are outcompeted by commensal organisms, and only become a medical concern when a healthy microbiome is damaged or thrown out of balance.

While on a space mission, astronauts do not get the same replenishment of microbes that they get on Earth. The traditional view of health microbiology is that microbes make you sick and should be removed from the spacecraft environment whenever possible. Astronauts' food is specially designed to last for long periods of time and has almost no microbial content (Cooper et al., 2011). To minimize the risk of infections, the air that astronauts breathe on space missions is heavily filtered, and everything shipped to the ISS is monitored to remove as many microorganisms as possible (Yamaguchi et al., 2014). While keeping an extremely clean spaceship environment has been an effective preventive measure for protecting astronauts from infections caused by many dangerous pathogens, the lack of microbial intake for long periods of time may have a detrimental effect on the diversity of the crew microbiota and, therefore, on astronaut health, to a sufficient extent that it may not be a viable strategy for long-term space missions.

While many impacts caused by microbiome imbalances, like diarrhea in Irritable Bowel Syndrome (IBS), are inconvenient and painful on Earth, they would be extremely distracting on space missions where astronauts need to stay healthy and focused on the mission objectives. A great deal of microbiome research is currently being conducted by NASA to determine how best to maintain microbiome homeostasis and astronaut health on future long-duration exploration missions.

\section{PAST AND CURRENT RESEARCH}

A significant amount of microbiology research has been done that spans the Apollo, Skylab, Space Shuttle, and International Space Station (ISS) programs. Until relatively recently, space microbiology research and monitoring consisted of growing microorganisms in petri dishes, and using microscopy and biochemical assays to determine what organism was present and what chemistry that particular organism was performing. Culture-based microbiology has the benefit of singling out an individual organism so that the observed chemistry can be attributed to that organism.

Identification of microbes associated with astronauts and space vehicles has been an active area of research, and reduction of pathogens from space missions has been a major priority from both a research and monitoring perspective (Yamaguchi et al., 2014). During the Apollo missions, the microbiomes of astronauts were classified in order to later recognize terrestrial contamination in lunar samples returned to Earth. Apollo astronauts were also examined to identify future microbial health hazards for the coming Skylab missions (Taylor, 1974). During the Skylab program, astronauts were methodically sampled 
before, during, and after missions, and it was found that while overall gastrointestinal (GI) microbial counts went up, diversity of the GI community went down following space flight. They also found an increase in certain pathogens like Serratia marcescens and Staphylococcus. aureus after space flight, and it was shown that $S$. aureus was transferred from astronaut to astronaut (Taylor et al., 1971), demonstrating that pathogens can be transmitted between crew members in the confined environment of a spaceship.

Traveling to space has been shown to reduce immune functioning in astronauts (Crucian et al., 2013, 2014; Cervantes and Hong, 2016) causing reactivation of latent viruses like herpes simplex virus 1, varicella zoster virus, and Epstein-Bar virus (Stowe et al., 2001; Mehta et al., 2013a,b, 2014) in astronauts on short- (weeks) and long- (months) duration missions. These viruses remain latent in humans and our immune systems usually keep them inactive, but they become reactivated in astronauts. That means that even if an opportunistic pathogen is dormant or present at trace, undetectable levels in an astronaut before they embark on a mission, reduced immune function may create a favorable environment for their reactivation or growth, becoming a potential health risk.

In recent years, advances in omics technologies have allowed researchers to perform genome-wide experiments to investigate the effects of space travel on microorganisms. These studies have demonstrated that several bacteria change their behavior, virulence, and gene expression under microgravity conditions. The opportunistic pathogen S. aureus, for instance, has been shown to form biofilms under simulated microgravity, causing a reduction in overall virulence and growth (Castro et al., 2011). Another opportunistic pathogen, Pseudomonas aeruginosa PAO1, was shown to change gene and protein expression under real or modeled microgravity, but it is unknown if these changes increase virulence of the pathogen (Crabbe et al., 2011). The fungal pathogen Candida albicans changed its budding configuration, gene expression, and cell aggregation while grown in space, although no enhancement of virulence was detected (Crabbe et al., 2013). Salmonella enterica serovar Typhimurium, on the other hand, was shown to increase virulence, cell counts in infected mice, and survival within a macrophage cell line when grown in simulated microgravity (Nickerson et al., 2000).

There are several drawbacks to culture-based microbiology, including the fact that the majority of known microbes cannot be grown in the lab. The exact percentage of microbes that can be cultured is still debated, but entire phyla of microbes have only been identified by marker gene studies with no cultured representatives (Handelsman, 2004). There is also the danger that isolating a microorganism that usually exists in a mixed community of organisms will cause it to change its behavior. Because of this, several cultureindependent methods of investigating microbial communities have been developed and are being applied to space microbiology research.

The Astronaut Microbiome project is a large study currently underway that seeks to employ culture-independent methods for studying the microbiome of astronauts, surfaces, and water at the ISS ${ }^{1}$. The project seeks to track the microbiome of astronauts before, during, and after ISS missions lasting from 6 months to 1 year using multiple techniques with the goal of measuring changes in the microbiome during space flight. The first technique involves sequencing of the $16 \mathrm{~s}$ ribosomal RNA gene, which is a marker that all microbial life possesses and allows identification of the microbes living in an environment. This approach provides a snapshot of bacteria present in an environment when the sample was taken. While this is helpful knowledge, 16s analysis does not provide accurate information about the biological functions performed by most of these microorganisms, making further investigation necessary. The second approach used by the project is metagenomic sequencing, which consists of sequencing all the DNA molecules present in an environment, and which determines the chemistry and metabolism that could be performed by the collection of genes encoded by the sequenced DNA molecules. Metagenomics is a more expensive and computationally intensive process, but it provides a more comprehensive knowledge about the chemical and metabolic potential of a microbial community compared to $16 \mathrm{~s}$ analysis. The Human Microbiome Project found that while the collection of microbial taxa may change among subjects from a particular healthy human cohort, the metabolic pathways represented by those organisms tend to remain relatively constant (Human Microbiome Project, 2012). Therefore, to evaluate how perturbations in the astronauts' microbiome promoted by space missions affect crew health, it is necessary not only to look at changes in microbial taxa through 16 s analysis, but also to carry out metagenomics studies to evaluate the impact of those changes on the metabolic capacity of the microbiome. Identification of variations in encoded microbial metabolic pathways during space flight will shed light into the impact of space travel on the microbiome and define what intervention, if any, is needed to maintain a balanced and healthy microbiome on long-duration exploration missions.

\section{FUTURE DIRECTIONS}

Methods for maintaining a balanced and healthy microbiome on long-term space missions will likely be a subject for ongoing research in the foreseeable future. Here we will address several areas of needed microbiome research as it relates to space travel. The first question is: How do we create environments in and around the astronauts that allow commensal organisms to flourish while still keeping pathogen concentrations as low as possible? And beyond that: How do we keep up a constant influx of commensal organisms to maintain the microbiomes of astronauts in homeostasis? How much damage will the microbiome sustain from Galactic Cosmic Radiation (GCR) once astronauts leave the Earth's Van Allen Belt? Finally, how will astronauts deal with bacterial infections that require antibiotic intervention and subsequent microbiome damage?

\footnotetext{
${ }^{1}$ https://lsda.jsc.nasa.gov/scripts/experiment/exper.aspx?exp_index =1836\&string $=$ microbiome \&CURRENT_STRING2=microbiome
} 


\section{Keeping Environmental Conditions That Favor the Growth and Maintenance of Healthy Bacteria in the Astronauts' Gut}

Prebiotics is a generic term used to describe dietary interventions that seek to create a healthy environment for the GI microbiome. Studies comparing the GI microbiome of tribal people, under high-fiber low-fat diets, to the microbiome of people from industrialized countries, consuming western diets rich in animal fats and poor in fiber, highlight the impact of diet on human health (Martinez et al., 2015; O'Keefe et al., 2015; Gomez et al., 2016). Indeed, these studies have shown that people following a western diet present a number of changes in their GI microbiota and metabolites that have been implicated in a number of human diseases, including cancer (Schwabe and Jobin, 2013; O'Keefe et al., 2015) and inflammatory bowel disease (Reif et al., 1997; Galvez et al., 2005). Typically, these changes involve a less diverse gut microbiota, characterized by the loss of major butyrate-producing taxa and several microbial metabolic pathways, such as butyrogenesis and saccharolytic fermentation, known to protect against inflammation and reduce cancer risk (Vital et al., 2014). Fortunately, these disease-associated features can be reverted in a relatively short period of time when switching to a low-fat high-fiber diet (O'Keefe et al., 2015).

Although still not conclusive, there is a wealth of evidence suggesting that space travel may lead to microbial dysbiosis and metabolic changes in the human gut, including a drop in alpha diversity and changes in gene expression of culturable bacteria. Also, reported dysregulation of the astronauts' immune system following short and long space flights, may be a consequence to in-flight alterations of the GI microbiota. It is likely that these negative effects might be potentially controlled or even prevented by diet-based therapies proven to promote a healthier GI microbial composition, such as those with a higher content of fiber (Turnbaugh et al., 2009; Zeng et al., 2014).

One way to add fiber to a diet is to increase the intake of fresh fruit and vegetables. To investigate whether this type of diet ameliorates the impact of space-travel on the immune system, gut microbial composition and overall astronaut's health, NASA has funded a project titled "Food Physiology" (Grace Douglas, 2016) which will seek to measure changes in the gut microbiome, immune system and metabolites when fresh fruit, vegetables, and fish (which contain omega 3 fatty acids) are added to an astronaut's diet. Fresh fruit and vegetables have the added benefit of association with healthy soil, one of the most microbially diverse environments we know of (Steinauer et al., 2015). While getting fresh fruit and vegetables to the ISS is an expensive but theoretically feasible proposition, it would only work on a mission to Mars if plants were gown in the spacecraft. Hydroponic gardens onboard space ships make for beautiful cinematography, but they create a host of issues for astronauts. Where do they get fresh soil? What do they do with the leftover plant material they do not eat? If they compost plant and astronaut waste, how do they keep humidity and fungus from spreading to the rest of the ship? Studies are currently underway at the ISS to better understand how to grow fruits and vegetables in space, but more research is necessary before space-grown food could make up a substantial portion of an astronaut's diet (Herridge, 2014; NASA ISS Program Science Office, 2016).

Another approach would be to try increasing the prebiotic qualities of space food, but that method also faces limitations. Food for a trip to Mars must be preserved for years and still be palatable. Astronauts will need to take food with them for the entire expedition, which places strict limits on the composition of the food (Cooper et al., 2011).

\section{Controlling the Influx of Commensal Organisms to Maintain the Microbiomes of Astronauts in Homeostasis}

It was mentioned earlier that microbial diversity in the GI tract of astronauts went down during the Skylab space missions (Taylor et al., 1971). There are likely several reasons for this phenomenon including nearly sterile meals and heavily filtered water and air. Astronauts aren't exposed to the same inputs of microorganisms as those present in terrestrial environments, and while transmission of bacteria from spacecraft to astronaut has been demonstrated (Taylor et al., 1971), it by no means makes up for the lack of incoming microbial diversity. There are several approaches that could address this shortfall of new microbial species. As previously discussed, growing fresh fruit and vegetables in-flight has many positive aspects but also comes with a host of logistical issues that would need to be worked out.

An alternative approach for introducing a constant input of microbial diversity to astronauts on long-duration missions would be prophylactic use of probiotics. Probiotics is a generic term used to describe dietary inputs of beneficial microbes. Probiotics can describe anything from yogurt with live cultures of bacteria and fungi, pills containing specific consortia of organisms, and even fecal transplants from healthy individuals. Humans have been eating probiotics in one form or another for much of our history, and the FDA labels many organisms as Generally Recognized as Safe, whose metabolic potential is unknown, but that humans have been consuming for many years without adverse health effects (Burdock and Carabin, 2004).

Use of probiotics would have several advantages, including ease of use, packaging, and storage, as well as being low on mass and space requirements, which will be issues on a trip to Mars. Further studies will be required to determine the most advantageous combination of microorganisms, as well as delivery mechanisms best suited for long-term stowage and stability during long-duration space missions ${ }^{2}$.

\section{Impact of Galactic Cosmic Radiation (GCR) on the Astronauts' Microbiome beyond the Earth's Van Allen Belt}

One question, which will need to be addressed before a human mission to Mars becomes a reality, is how to deal with GCR and, specifically, any potential damage it may cause to the

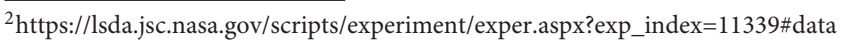


microbiome. Astronauts on a mission to Mars will encounter low dosages of high-energy radiation that is ubiquitous outside of the Earth's Van Allen Belt (Badhwar and O'Neill, 1996). Astronauts at the ISS are shielded from the full effects of GCR because the orbit of the ISS is still inside the Van Allen Belt, meaning researchers have a limited knowledge about the effects of constant low-dosage radiation on the crew microbiome. Most microbes are not resistant to radiation, and studies that have investigated the effect of higher doses of radiation on Earth have demonstrated that radiation can cause substantial damage to the microbiome (Packey and Ciorba, 2010; Lam et al., 2012). To further complicate matters, GCR could potentially damage most microbes in the spacecraft, including those on and in the astronaut body as well as those from probiotic stocks. Further research will need to address potential GCR damage to the microbiome so that more complete measures of risk to the astronaut's health can be determined.

\section{Antibiotic Usage and Microbial Dysbiosis}

Currently, the recommended countermeasure to deal with bacterial infections in the ISS is the administration of antibiotics. Antibiotics have been an effective method for treating bacterial infections for many years. While antibiotics will effectively remove most bacterial infections, they will also do substantial damage to the commensal members of the GI microbiome (Preidis and Versalovic, 2009). This can cause a range of issues, including pathogenic colonization of Clostridium difficile, which can cause chronic GI issues and extreme diarrhea (Kelly et al., 1994; Theriot et al., 2014). In normal conditions, C. difficile is usually present at low levels in the human GI tract, mostly due to competitive displacement by commensal GI microbes. However, $C$. difficile has been found to colonize the GI tract when commensal GI microbial communities are damaged by antibiotics (Kelly et al., 1994).

Probiotics constitute an excellent option to help replenish the GI microbiome during and after antibiotic treatment, and they are currently used clinically to treat $C$. difficile infections that are resistant to antibiotics (McFarland, 2006; Szajewska et al., 2006; Avadhani and Miley, 2011; Hempel et al., 2012; Pattani et al., 2013). One potential issue for long-duration space missions is that probiotics provide only a limited selection of commensal GI organisms. On Earth, probiotic use is supplemented by a healthy influx of organisms from food, water, and air; these are sources of commensal organisms not currently available to astronauts in-flight.

One option for emergency in-flight antibiotic use may be to package astronauts' stool into probiotic capsules while they are healthy on Earth. This would allow an astronaut to refresh their GI microbiome with a diverse set of organisms from their

\section{REFERENCES}

Avadhani, A., and Miley, H. (2011). Probiotics for prevention of antibioticassociated diarrhea and Clostridium difficile-associated disease in hospitalized adults-a meta-analysis. J. Am. Acad. Nurse Pract. 23, 269-274. doi: 10.1111/j.1745-7599.2011.00617.x own healthy GI tract. Regardless of the source, one or more methods of rebalancing a damaged microbiome will be essential for long-duration space missions.

\section{CONCLUSION}

Extensive research into the maintenance of a healthy microbiome of astronauts on space missions has been a topic of research for decades, and further studies are currently underway. These studies are starting to reveal that space travel alters the composition and physiology of the astronauts' gut microbiome. In the long run, compositional changes in the gut flora, in combination with an already weakened immune response, might pave the way for infections by opportunistic pathogens or predispose astronauts to a number of diseases such as IBS or even cancer. This scenario could be made worse by long-term exposure to the extremely clean environment of the spacecraft and the potential deleterious effect of GCR on the astronauts' microbiota, and therefore, the risks associated with these factors should be explored in more depth.

Diet based therapies, such as the incorporation of fresh fruit and vegetables, are a promising countermeasure to deal with health risks associated with microbial dysbiosis in space. Fiberrich diets promote the growth of healthy butyrate-producing bacteria in the gut and help to revert metabolite-profiles associated with human diseases. Although space farming is still in its infancy, the Food Physiology project at NASA will use a combination of metabolomics, metatranscriptomics, 16s taxonomic profiling and immunological approaches to directly test the hypothesis that diets rich in fiber are able to reverse the detrimental effects of space travel on the human microbiome and immune response.

Probiotics are also a promising alternative to fight microbial dysbiosis in space, particularly in situations where the GI flora has been significantly depleted of its original microbial content, for example following antibiotic treatment. Further research will be needed to determine whether commercially available probiotics containing just a small number of beneficial bacteria species or fecal transplant pills are the more effective therapy for restoring an altered gut microbiome.

\section{AUTHOR CONTRIBUTIONS}

AV and HL wrote and edited the manuscript.

\section{FUNDING}

This work was funded by NASA grant NNX12AB02G. 
Burdock, G. A., and Carabin, I. G. (2004). Generally recognized as safe (GRAS): history and description. Toxicol. Lett. 150, 3-18. doi: 10.1016/j.toxlet.2003.07.004

Castro, S. L., Nelman-Gonzalez, M., Nickerson, C. A., and Ott, C. M. (2011). Induction of attachment-independent biofilm formation and repression of $\mathrm{Hfq}$ expression by low-fluid-shear culture of Staphylococcus aureus. Appl. Environ. Microbiol. 77, 6368-6378. doi: 10.1128/AEM.00175-11

Cervantes, J. L., and Hong, B. Y. (2016). Dysbiosis and immune dysregulation in outer space. Int. Rev. Immunol. 35, 67-82. doi: 10.3109/08830185.2015.1027821

Consortium, H. M. P. (2012). Structure, function and diversity of the healthy human microbiome. Nature 486, 207-214. doi: 10.1038/Nature11234

Cooper, M., Douglas, G., and Perchonok, M. (2011). Developing the NASA food system for long-duration missions. J. Food Sci. 76, R40-R48. doi: $10.1111 / \mathrm{j} .1750-3841.2010 .01982 . \mathrm{x}$

Costello, E. K., Lauber, C. L., Hamady, M., Fierer, N., Gordon, J. I., and Knight, R. (2009). Bacterial community variation in human body habitats across space and time. Science 326, 1694-1697. doi: 10.1126/science.1177486

Crabbe, A., Nielsen-Preiss, S. M., Woolley, C. M., Barrila, J., Buchanan, K., McCracken, J., et al. (2013). Spaceflight enhances cell aggregation and random budding in Candida albicans. PLoS ONE 8:e80677. doi: 10.1371/journal.pone.0080677

Crabbe, A., Schurr, M. J., Monsieurs, P., Morici, L., Schurr, J., Wilson, J. W., et al. (2011). Transcriptional and proteomic responses of pseudomonas aeruginosa PAO1 to spaceflight conditions involve hfq regulation and reveal a role for oxygen. Appl. Environ. Microbiol. 77, 1221-1230. doi: 10.1128/Aem. 01582-10

Crucian, B., Stowe, R., Mehta, S., Uchakin, P., Quiriarte, H., Pierson, D., et al. (2013). Immune system dysregulation occurs during short duration spaceflight on board the space shuttle. J. Clin. Immunol. 33, 456-465. doi: 10.1007/s10875012-9824-7

Crucian, B. E., Zwart, S. R., Mehta, S., Uchakin, P., Quiriarte, H. D., Pierson, D., et al. (2014). Plasma cytokine concentrations indicate that in vivo hormonal regulation of immunity is altered during long-duration spaceflight. J. Interferon Cytokine Res. 34, 778-786. doi: 10.1089/jir.2013.0129

David, L. A., Materna, A. C., Friedman, J., Campos-Baptista, M. I., Blackburn, M. C., Perrotta, A., et al. (2014a). Host lifestyle affects human microbiota on daily timescales. Genome Biol. 15:R89. doi: 10.1186/gb-2014-15-7-r89

David, L. A., Maurice, C. F., Carmody, R. N., Gootenberg, D. B., Button, J. E., Wolfe, B. E., et al. (2014b). Diet rapidly and reproducibly alters the human gut microbiome. Nature 505, 559-563. doi: 10.1038/nature12820

Douglas, G. (2016). The Integrated Impact of Diet on Human Immune Response, the Gut Microbiota, and Nutritional Status During Adaptation to Spaceflight. Available online at: https://humanresearchroadmap.nasa.gov/tasks/task.aspx? $\mathrm{i}=2068$

Galvez, J., Rodriguez-Cabezas, M. E., and Zarzuelo, A. (2005). Effects of dietary fiber on inflammatory bowel disease. Mol. Nutr. Food Res. 49, 601-608. doi: $10.1002 / \mathrm{mnfr} .200500013$

Giongo, A., Gano, K. A., Crabb, D. B., Mukherjee, N., Novelo, L. L., Casella, G., et al. (2011). Toward defining the autoimmune microbiome for type 1 diabetes. Isme J. 5, 82-91. doi: 10.1038/ismej.2010.92

Gomez, A., Petrzelkova, K. J., Burns, M. B., Yeoman, C. J., Amato, K. R., Vlckova, K., et al. (2016). Gut microbiome of coexisting baaka pygmies and bantu reflects gradients of traditional subsistence patterns. Cell Rep. 14, 2142-2153. doi: 10.1016/j.celrep.2016.02.013

Handelsman, J. (2004). Metagenomics: application of genomics to uncultured microorganisms. Microbiol. Mol. Biol. Rev. 68, 669-685. doi: 10.1128/MMBR.68.4.669-685.2004

Hanski, I., von Hertzen, L., Fyhrquist, N., Koskinen, K., Torppa, K., Laatikainen, T., et al. (2012). Environmental biodiversity, human microbiota, and allergy are interrelated. Proc. Natl. Acad. Sci. U.S.A. 109, 8334-8339. doi: 10.1073/pnas. 1205624109

Hempel, S., Newberry, S. J., Maher, A. R., Wang, Z., Miles, J. N., Shanman, R., et al. (2012). Probiotics for the prevention and treatment of antibiotic-associated diarrhea: a systematic review and meta-analysis. JAMA 307, 1959-1969. doi: 10.1001/jama.2012.3507

Herridge, L. (2014). Veggie Plant Growth System Activated on International Space Station. Available online at: http://www.nasa.gov/content/veggie-plantgrowth-system-activated-on-international-space-station
Human Microbiome Project, C. (2012). Structure, function and diversity of the healthy human microbiome. Nature 486, 207-214. doi: 10.1038/nature11234

Kelly, C. P., Pothoulakis, C., and LaMont, J. T. (1994). Clostridium difficile colitis. N. Engl. J. Med. 330, 257-262. doi: 10.1056/NEJM199401273300406

Lam, V., Moulder, J. E., Salzman, N. H., Dubinsky, E. A., Andersen, G. L., and Baker, J. E. (2012). Intestinal microbiota as novel biomarkers of prior radiation exposure. Radiat. Res. 177, 573-583. doi: 10.1667/Rr2691.1

Luna, R. A., and Foster, J. A. (2014). Gut brain axis: diet microbiota interactions and implications for modulation of anxiety and depression. Curr. Opin. Biotechnol. 32C, 35-41. doi: 10.1016/j.copbio.2014.10.007

Martinez, I., Stegen, J. C., Maldonado-Gomez, M. X., Eren, A. M., Siba, P. M., Greenhill, A. R., et al. (2015). The gut microbiota of rural papua new guineans: composition, diversity patterns, and ecological processes. Cell Rep. 11, 527-538. doi: 10.1016/j.celrep.2015.03.049

McFarland, L. V. (2006). Meta-analysis of probiotics for the prevention of antibiotic associated diarrhea and the treatment of Clostridium difficile disease. Am. J. Gastroenterol. 101, 812-822. doi: 10.1111/j.1572-0241.2006.00465.x

Mehta, S. K., Crucian, B. E., Stowe, R. P., Simpson, R. J., Ott, C. M., Sams, C. F., et al. (2013a). Reactivation of latent viruses is associated with increased plasma cytokines in astronauts. Cytokine 61, 205-209. doi: 10.1016/j.cyto.2012.09.019

Mehta, S. K., Laudenslager, M. L., Stowe, R. P., Crucian, B. E., Sams, C. F., and Pierson, D. L. (2014). Multiple latent viruses reactivate in astronauts during Space Shuttle missions. Brain Behav. Immun. 41, 210-217. doi: 10.1016/j.bbi.2014.05.014

Mehta, S. K., Tyring, S. K., Cohrs, R. J., Gilden, D., Feiveson, A. H., Lechler, K. J., et al. (2013b). Rapid and sensitive detection of varicella zoster virus in saliva of patients with herpes zoster. J. Virol. Methods 193, 128-130. doi: 10.1016/j.jviromet.2013.05.019

NASA ISS Program Science Office (2016). Vegetable Production System (Veggie) 01.13.16. Retrieved from http://www.nasa.gov/mission_pages/station/research/ experiments/383.html

Nickerson, C. A., Ott, C. M., Mister, S. J., Morrow, B. J., Burns-Keliher, L., and Pierson, D. L. (2000). Microgravity as a novel environmental signal affecting Salmonella enterica serovar Typhimurium virulence. Infect. Immun. 68, 3147-3152. doi: 10.1128/Iai.68.6.3147-3152.2000

Nickerson, C. A., Ott, C. M., Wilson, J. W., Ramamurthy, R., and Pierson, D. L. (2004). Microbial responses to microgravity and other low-shear environments. Microbiol. Mol. Biol. Rev. 68, 345-361. doi: 10.1128/MMBR.68.2.345-361.2004

O'Keefe, S. J., Li, J. V., Lahti, L., Ou, J., Carbonero, F., Mohammed, K., et al. (2015). Fat, fibre and cancer risk in African Americans and rural Africans. Nat. Commun. 6:6342. doi: 10.1038/ncomms7342

Packey, C. D., and Ciorba, M. A. (2010). Microbial influences on the small intestinal response to radiation injury. Curr. Opin. Gastroenterol. 26, 88-94. doi: 10.1097/MOG.0b013e3283361927

Pattani, R., Palda, V. A., Hwang, S. W., and Shah, P. S. (2013). Probiotics for the prevention of antibiotic-associated diarrhea and Clostridium Difficile infection among hospitalized patients: systematic review and meta-analysis. Open Med. 7, e56-e67.

Preidis, G. A., and Versalovic, J. (2009). Targeting the human microbiome with antibiotics, probiotics, and prebiotics: gastroenterology enters the metagenomics era. Gastroenterology 136, 2015-2031.

Reif, S., Klein, I., Lubin, F., Farbstein, M., Hallak, A., and Gilat, T. (1997) Pre-illness dietary factors in inflammatory bowel disease. Gut 40, 754-760.

Saulnier, D. M., Riehle, K., Mistretta, T. A., Diaz, M. A., Mandal, D., Raza, S., et al. (2011). Gastrointestinal microbiome signatures of pediatric patients with irritable bowel syndrome. Gastroenterology 141, 1782-1791. doi: 10.1053/j.gastro.2011.06.072

Schwabe, R. F., and Jobin, C. (2013). The microbiome and cancer. Nat. Rev. Cancer 13, 800-812. doi: $10.1038 / \mathrm{nrc} 3610$

Steinauer, K., Tilman, D., Wragg, P. D., Cesarz, S., Cowles, J. M., Pritsch, K., et al. (2015). Plant diversity effects on soil microbial functions and enzymes are stronger than warming in a grassland experiment. Ecology 96, 99-112. doi: 10.1890/14-0088.1

Stowe, R. P., Mehta, S. K., Ferrando, A. A., Feeback, D. L., and Pierson, D. L. (2001). Immune responses and latent herpesvirus reactivation in spaceflight. Aviat. Space Environ. Med. 72, 884-891.

Szajewska, H., Ruszczynski, M., and Radzikowski, A. (2006). Probiotics in the prevention of antibiotic-associated diarrhea in children: a 
meta-analysis of randomized controlled trials. J. Pediatr. 149, 367-372. doi: 10.1016/j.jpeds.2006.04.053

Taylor, G. R., Graves, R. C., Brockett, R. M., Ferguson, J. K., and Mieszkuc, B. J. (1971). Skylab Environmental and Crew Microbiology Studies. Washington, United States: NASA Johnson Space Center; Houston, TX, United States. Available online at: http://ntrs.nasa.gov/search.jsp?R=19770026836

Taylor, G. R. (1974). Space microbiology. Annu. Rev. Microbiol. 28, 121-137. doi: 10.1146/annurev.mi.28.100174.001005

Theriot, C. M., Koenigsknecht, M. J., Carlson, P. E. Jr., Hatton, G. E., Nelson, A. M., Li, B., et al. (2014). Antibiotic-induced shifts in the mouse gut microbiome and metabolome increase susceptibility to Clostridium difficile infection. Nat. Commun. 5:3114. doi: 10.1038/ncomms4114

Turnbaugh, P. J., Ridaura, V. K., Faith, J. J., Rey, F. E., Knight, R., and Gordon, J. I. (2009). The effect of diet on the human gut microbiome: a metagenomic analysis in humanized gnotobiotic mice. Sci. Transl. Med. 1:6ra14. doi: 10.1126/scitranslmed.3000322

Vital, M., Howe, A. C., and Tiedje, J. M. (2014). Revealing the bacterial butyrate synthesis pathways by analyzing (meta)genomic data. mBio 5:e00889. doi: 10.1128/mBio.00889-14

Wilson, J. W., Ott, C. M., Quick, L., Davis, R., Honer zu Bentrup, K., Crabbe, A., et al. (2008). Media ion composition controls regulatory and virulence response of Salmonella in spaceflight. PLoS ONE 3:e3923. doi: 10.1371/journal.pone.0003923

Yamaguchi, N., Roberts, M., Castro, S., Oubre, C., Makimura, K., Leys, N., et al. (2014). Microbial monitoring of crewed habitats in spacecurrent status and future perspectives. Microbes Environ. 29, 250-260. doi: 10.1264/jsme2.ME14031

Zeng, H., Lazarova, D. L., and Bordonaro, M. (2014). Mechanisms linking dietary fiber, gut microbiota and colon cancer prevention. World J. Gastrointest. Oncol. 6, 41-51. doi: 10.4251/wjgo.v6. i2.41

Conflict of Interest Statement: The authors declare that the research was conducted in the absence of any commercial or financial relationships that could be construed as a potential conflict of interest.

Copyright (c) 2016 Voorhies and Lorenzi. This is an open-access article distributed under the terms of the Creative Commons Attribution License (CC BY). The use, distribution or reproduction in other forums is permitted, provided the original author(s) or licensor are credited and that the original publication in this journal is cited, in accordance with accepted academic practice. No use, distribution or reproduction is permitted which does not comply with these terms. 